\title{
A single-centre experience of intravenous thrombolysis for stroke in COVID-19 patients
}

\author{
Davide Sangalli ${ }^{1}$ (D) Valeria Polonia ${ }^{1,2} \cdot$ Daniele Colombo $^{1} \cdot$ Vittorio Mantero ${ }^{1} \cdot$ Marco Filizzolo $^{3}$. \\ Chiara Scaccabarozzi ${ }^{1} \cdot$ Andrea Salmaggi $^{1}$
}

Received: 2 June 2020 / Accepted: 8 July 2020 / Published online: 12 July 2020

(C) Fondazione Società Italiana di Neurologia 2020

\begin{abstract}
The sudden worldwide outbreak of Coronavirus Disease 2019 (COVID-19) has certainly provided new challenges in the management of acute ischaemic stroke, and the risk-benefit ratio of intravenous thrombolysis in COVID-19 positive patients is not well known. We describe four COVID-19 patients treated with intravenous thrombolysis for acute ischaemic stroke. Although rt-PA administration is the main therapeutic strategy, our patients experienced unpredictable complications and showed atypical features: the overall mortality was very high. In conclusion, in this article, we provide information about these cases and discuss the possible explanation behind this trend.
\end{abstract}

Keywords Stroke $\cdot$ Intravenous thrombolysis $\cdot$ rt-PA $\cdot$ COVID-19

\section{Introduction}

The sudden worldwide outbreak of Coronavirus Disease 2019 (COVID-19) has certainly provided new challenges in the management of acute ischaemic stroke. In Italy, some hospitals have been promptly switched into COVID-19 centres and stroke patients diverted to other designated stroke units. In these hubs, a higher number of intravenous thrombolysis or mechanical thrombectomy procedures have been performed in both coronavirus positive and negative patients. Moreover, COVID-19 itself increases the risk of thromboembolic events without any scientific evidence on the best management [1]. Current guidelines recommend intravenous thrombolysis as the first-line therapy in eligible patients. Excluding infectious endocarditis, no other infectious disease is a contraindication to intravenous recombinant

Davide Sangalli

d.sangalli@asst-lecco.it

1 Unit of Neurology, “Alessandro Manzoni” Hospital - ASST Lecco, Via dell'Eremo 9/11, 23900 Lecco, Italy

2 University of Milano-Bicocca, Milan, Italy

3 Unit of Neuroradiology, “Alessandro Manzoni” Hospital - ASST Lecco, Lecco, Italy tissue plasminogen activator (rt-PA) [2]. Nevertheless, sepsis and milder inflammatory response influence negatively the rate of complications, disability and death in stroke patients [3].

In this communication, we describe our preliminary experience of a Stroke Hub in Lombardia (Italy) in treating COVID-19 patients with intravenous thrombolysis in acute ischaemic stroke.

\section{Case 1}

A 75-year-old woman with a history of hypertension, dyslipidaemia and chronic kidney disease was admitted for acute onset of global aphasia and right hemiparesis. She was diagnosed with acute ischaemic stroke and was given intravenous rt-PA. No major arterial occlusion was detected. She was COVID-19 positive; her husband had died of COVID-19 pneumonia 3 days before. The patient experienced an initial improvement of right hemiparesis, but a second brain CT scan showed a left frontotemporal and parietal ischaemic lesion complicated by haemorrhagic transformation. During hospitalization, atrial fibrillation was detected. She was persistently feverish and finally developed respiratory insufficiency, treated with high flow oxygen therapy, piperacillin/tazobactam and Darunavir. Despite treatments, she deceased a week later. 


\section{Case 2}

A 53-year-old man with a history of hypertension and dyslipidaemia developed acute left sensorimotor hemisyndrome: intravenous thrombolysis for acute ischaemic stroke was administered. We excluded the indication for mechanical thrombectomy with a CT angiography. In the previous days, he had experienced cough and myalgias. Chest CT scan showed ground glass opacities and nasopharyngeal swab was positive for low levels of SARS-CoV-2 RNA. The patient did not show any clinical sign of pneumonia and was treated with hydroxychloroquine alone. The deficits progressively improved and he was eventually discharged with a normal neurological and pulmonary clinical examination.

\section{Case 3}

A 77-year-old diabetic patient was admitted for wake-up onset of global aphasia and mild right hemiparesis. We performed brain CT scan with perfusion, showing a complete CBV/MTT mismatch without early ischaemic changes. He was given intravenous rt-PA. No endovascular treatment was performed. Chest CT scan showed COVID-19 related findings: he was administered hydroxychloroquine and antibiotic therapy. The next day, CT scan revealed a malignant haemorrhagic transformation of his left-hemisphere ischaemic stroke. Concurrently, the patient developed an EKG ST depression with cardiac troponin increase. He was diagnosed with subendocardial acute ischaemia, confirmed with a transthoracic echocardiography. He rapidly entered a comatose state and died 3 days later.

\section{Case 4}

A 59-year old man with a biological aortic valve prosthesis presented with acute-onset aphasia. He had a 3-week history of fever and cough. His general practitioner had clinically diagnosed COVID-19, treated with hydroxychloroquine and azithromycin. At admission, on suspicion of ischaemic stroke, in absence of contraindications, we started intravenous thrombolysis. During the infusion, the patient developed massive epistaxis and oral bleeding, so the infusion was stopped (more than $85 \%$ of the total rt-PA dose had already been administered).

Despite the resolution of the language disorder after thrombolysis, a control brain CT scan showed bilateral both ischaemic and haemorrhagic lesions. A chest CT scan revealed COVID-19 related pneumonia, treated with antivirals, antibiotics and oxygen therapy.

Transthoracic echocardiography showed infective endocarditis of the biological valve with a periprosthetic abscess.
Cultures on blood specimens showed growth of Enterococcus faecalis, with response to ampicillin and ceftriaxone. Ten days later, brain CT scan showed reabsorption of haemorrhagic foci with neurological complete improvement. The patient was scheduled for cardiac surgery. Unfortunately, he never underwent surgery, because he had a sudden cardiac arrest a few days later. Since he had COVID-19, according to our hospital rules, we could not order an autopsy to certify the cause of death.

\section{Discussion}

To our knowledge, only one case of intravenous thrombolysis for stroke in a COVID-19 patient has been reported [4]. Although rt-PA administration and endovascular treatment remain the core therapeutic strategies, atypical features and complications characterized the patients with SARS-COV-2 [5].

A worse clinical outcome in patients with stroke and concomitant infection is well-known [3]. This seems to be the same in patients presenting with stroke in the context of COVID-19: the overall mortality rate has been $38.5 \%$ in a small cohort of patients with COVID-19 developing stroke [6]. In our hospital, during the month of March and April, the overall mortality of patients with ischaemic stroke and COVID-19 is $50 \%$ (3/4 in the patients treated with rt-PA and $4 / 10$ in the others). During the same 2-month period, mortality in the COVID-19 negative group with ischaemic stroke was significantly lower i.e. $12 \%(3 / 15$ in the patients treated with rt-PA or mechanical thrombectomy and $3 / 35$ in the non-treated group).

In our four patients, cause of death was not always easy to assess reliably due to pandemic-induced constraints in availability of diagnostic facilities.

Of note, the only patient who survived displayed mildly elevated CRP levels and normal D-dimer values at admission and no haemorrhagic complication, while all the other three patients displayed elevated inflammatory indices and bleeding abnormalities (Table 1).

In conclusion, although the risk-benefit ratio in stroke patients treated with thrombolytic agents in times of COVID-19 pandemic is not well known, there are still no laboratory data or COVID-19 related changes that formally contraindicate the administration of rt-PA [7, 8].

Ongoing collaborative efforts are collecting more data from various centres that hopefully will help clinicians to better delineate the risk/benefit profile of reperfusion strategies in patients with stroke and COVID-19 infection; pending these results, clinicians should be aware of the non-negligible risk inherent to rt-PA treatment delivered to COVID-19 stroke patients. 


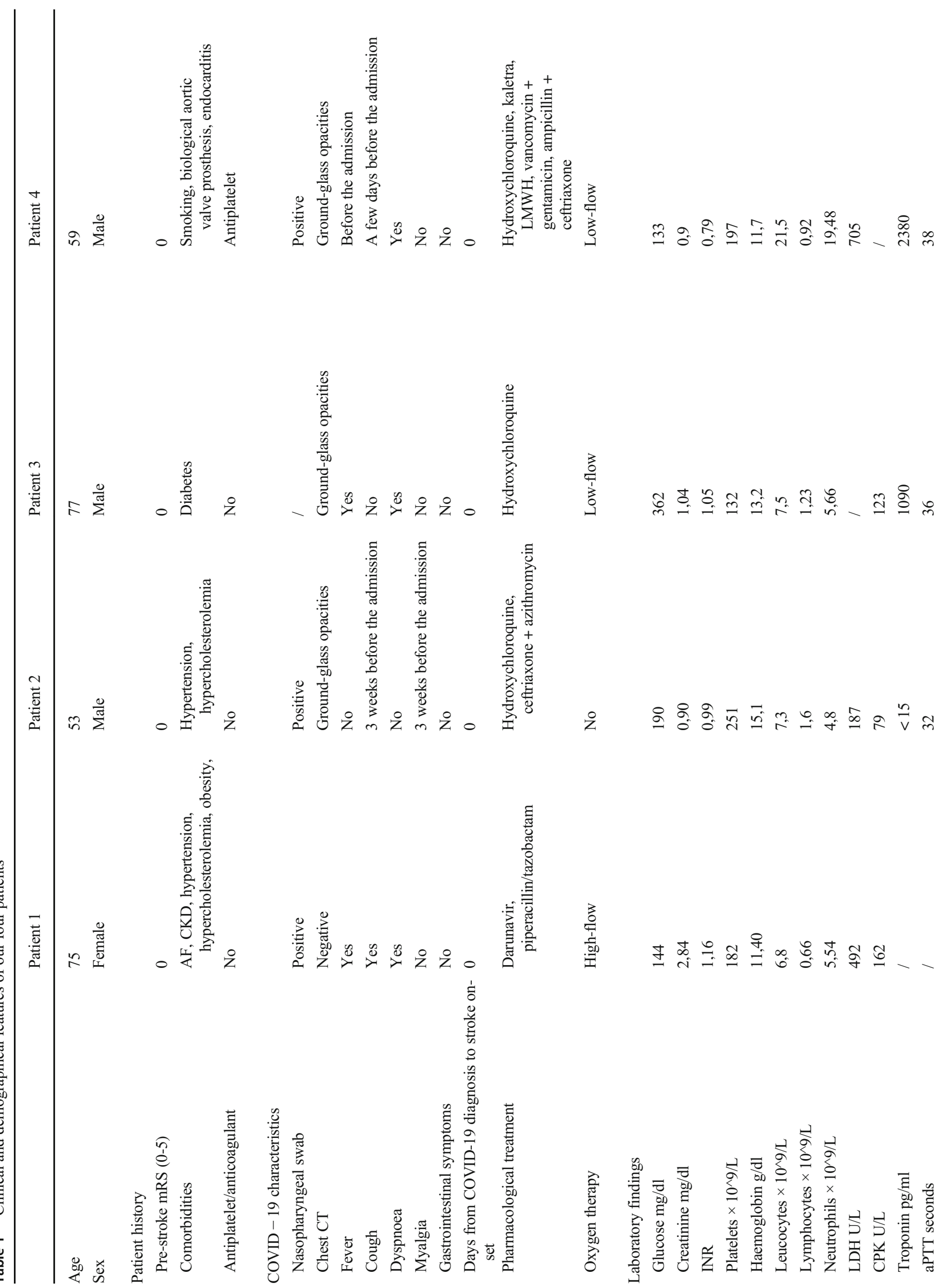




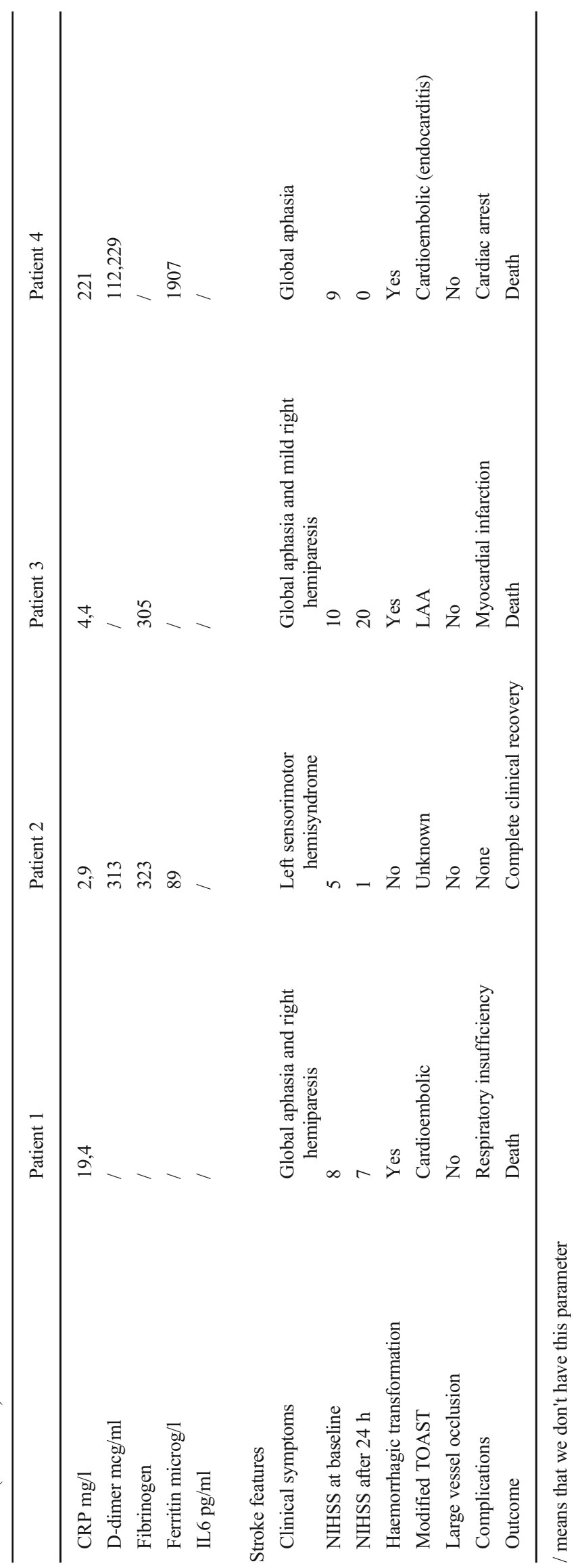




\section{Compliance with ethical standards}

Conflict of interest The authors declare that they have no conflict of interest.

Ethical approval All procedures performed in studies involving human participants were in accordance with the ethical standards of the institutional and/or national research committee and with the 1964 Helsinki Declaration and its later amendments or comparable ethical standards.

Informed consent Informed consent was obtained from all individual participants included in the study.

\section{References}

1. Zhou F, Yu T, Du R et al (2020) Clinical course and risk factors for mortality of adult inpatients with COVID-19 in Wuhan, China: a retrospective cohort study. Lancet 395(10229):1054-1062

2. Powers WJ, Rabinstein AA, Ackerson T, Adeoye OM, Bambakidis NC, Becker K, Biller J, Brown M, Demaerschalk BM, Hoh B, Jauch EC, Kidwell CS, Leslie-Mazwi TM, Ovbiagele B, Scott PA, Sheth KN, Southerland AM, Summers DV, Tirschwell DL (2019) Guidelines for the early management of patients with acute ischemic stroke: 2019 update to the 2018 guidelines for the early management of acute ischemic stroke: a guideline for healthcare professionals from the American Heart Association/American Stroke Association. Stroke 50:e344-e418

3. Helsinki Stroke Thrombolysis Registry Group (2013) Body temperature, blood infection parameters, and outcome of thrombolysistreated ischemic stroke patients. Int J Stroke 8:632-638

4. Co COC, Yu JRT, Laxamana LC, David-Ona DIA (2020) Intravenous thrombolysis for stroke in a COVID-19 positive filipino patient, a case report. J Clin Neurosci:S0967-5868(20)30727-X

5. Mao L, Wang M, Chen S, He Q, Chang J, Hong C, et al. Neurological manifestations of hospitalized patients with COVID19 in Wuhan, China: a retrospective case series study. medRxiv 2020.02.22.20026500, https://doi.org/10.1101/2020.02.22. 20026500 (accessed 17 Apr 2020)

6. Li Y., Wang M., Zhou Y. et al. Acute cerebrovascular disease following COVID-19: a single-center, retrospective, observational study (3/3/2020), https://ssrn.com/abstract1/43550025 or https://doi. org/10.2139/ssrn.3550025 (accessed 17 Apr 2020)

7. Wang J, Hajizadeh N, Moore EE, McIntyre RC, Moore PK, Veress LA, Yaffe MB, Moore HB, Barrett CD (2020) Tissue plasminogen activator (tPA) treatment for COVID-19 associated acute respiratory distress syndrome (ARDS): a case series. Thromb Haemost 18: 1752-1755. https://doi.org/10.1111/jth.14828

8. Hess DC, Eldahshan W, Rutkowski E (2020 Jun) COVID-19-related stroke. Transl Stroke Res 11(3):322-325. https://doi.org/10.1007/ s12975-020-00818-9

Publisher's note Springer Nature remains neutral with regard to jurisdictional claims in published maps and institutional affiliations. 